\title{
Informed consent and BRCA1 testing
}

Sir - The report by Struewing et al. in Nature Genetics that the 185delAG mutation in the BRCA1 gene occurs in about $1 \%$ of Ashkenazi Jews ${ }^{1}$ follows recent studies that report considerable interest in genetic testing for susceptibility to breast cancer among women at varying levels of risk. In one study of almost 1,000 mammography and gynecology patients, $90 \%$ in both groups said they would take such a test $^{2}$. In another study of 105 firstdegree relatives of breast cancer patients, $91 \%$ said they would want to be tested ${ }^{3}$. In that study, the primary reason that women gave for wanting to be tested was to learn their children's risk. Unfortunately, despite their acknowledgement of the importance of educating participants about $B R C A 1$ testing, these studies included either limited or no education.

Our research suggests that when women are given information about the test itself, and not merely about its availability, their interest in testing wanes. Furthermore, what motivates women to undergo testing may be broader than simply learning about their own or their children's risk. The discovery of the 185delAG mutation in Ashkenazi Jews highlights the urgent need for informed consent protocols that include both educational and decision-making components.

As part of our effort to develop a model informed consent process for $B R C A 1$ testing, we conducted nine focus groups of women $(n=86)$, stratified by socioeconomic status (SES), race and breast cancer risk. The women were recruited through advertisements in local newspapers, and offered $\$ 50$ for their participation. The purpose of the focus groups was to obtain a better sense of women's understanding of testing, what they would want to know about testing if it were offered, whether they would be interested in testing, their reasons for such interest, and the process by which they would make a decision about the test.

Knowledge and concerns varied by demographic characteristics and risk. Women in the general population who were of lower SES were both less knowledgeable about breast cancer and more distrustful of the health care system than other groups of women. Moreover, there were racial differences in the nature of that distrust. White women thought that breast cancer screening was a way for the medical establishment to make money; African-American women feared possible exploitation if they participated in (any kind of) research. When asked what they would want to know about $B R C A 1$ testing if they were to be offered it, women in high SES groups requested information on the validity and accuracy of the test, cost of testing, follow-up recommendations and implications of test results for other family members. Women in low SES groups were more concerned about practical aspects of testing, such as what testing involved (blood samples and so on), who would do the test, when the results would be available, and if the test detected other cancers.

Regardless of SES, participants were very interested in $B R C A 1$ testing until the limitations and uncertainties associated with the test were understood. In particular, when they learned that most breast cancer is not associated with a BRCA1 mutation, that effective means of preventing breast cancer have not been proven, and that there are risks associated with disclosing test results to employers and insurers, participants began to question the value of testing. Participants were not generally in favour of prenatal or childhood testing either for themselves or for others. Our findings are consistent with the hypothesis (and anecdotal experience of our clinical coauthors) that, when women understand the benefits and limitations of testing for genetic susceptibility, their interest in testing decreases. In our sample, this was true of high-risk women as well as women in the general population.

We recently conducted a tenth focus group, this one of Jewish women, to understand the degree to which the news about the 185delAG mutation would influence their interest in testing. In contrast to the other groups, this group did not lose interest in testing after receiving information. This seemed to be explained not by an inadequate understanding of the factual information that was presented to them, but by their perceived social obligation to do anything they could to advance medical science. They would be interested in testing in the context of research, even if their individual results were withheld. In short, these women did not perceive any dis- advantages of participating in a testing protocol, and would seek testing for reasons other than determining their own susceptibility.

We will be attempting to quantify these qualitative findings in a subsequent survey of both women at increased genetic risk for breast cancer and those in the general population. However, more qualitative work needs to be done as well. We need to understand whether altruism, trust or distrust in the medical establishment and the desire to seek personal genetic information for the sake of one's children function as barriers to truly autonomous decision-making. Until these issues are addressed, those obtaining consent from African-American women should be sensitive to the general distrust of health services, many of which may be valuable to them, while those obtaining consent from Jewish women should be aware of the 'slippery slope' from perceived social responsibility to coercion.

More generally, any evidence of substantial interest in genetic susceptibility testing in the absence of efforts to educate women about limitations as well as benefits should be interpreted very cautiously ${ }^{2,3}$. Even with education, we know that interest in testing is distinct from participation in testing $^{4,5}$. Moreover, our findings suggest that informed consent for participation in research, as well as for testing in clinical practice, should not only focus on educating prospective participants. It should include a decision-making component that explores women's understanding, their perceptions of barriers to testing and their reasons for and against testing.

\section{Gail Geller}

Barbara A. Bernhardt

Kathy Helzlsouer

Neil A. Holtzman

Michael Stefanek

Patti M. Wilcox

Genetics \& Public Policy Studies,

Johns Hopkins School of Medicine,

Baltimore, Maryland 21205-2004, USA

1. Struewing, J.P. et al. Nature Genet. 11, 1-3(1995),

2. Chaliki, H. et al. Am. J. Pub. Hith. $85,1133-1135$ (1995).

3. Leman, C., Seay, J., Balshem, A. \& Audrain, J.Am. J. med. Genet. 57, 385-392 (1995).

4. Tembor, E.S. et al. Am. J. hum. Genet. 55, 626-637 (1994).

5. Cuaid, K.A. \& Moris, M. Am. J. med. Genet. 45 $41-45(1993)$. 